\title{
THE PHASE PROBLEM OF ULTRAFLAT UNIMODULAR POLYNOMIALS: THE RESOLUTION OF THE CONJECTURE OF SAFFARI
}

\section{TAMÁS ERDÉLYI}

Abstract. Let $D$ be the open unit disk of the complex plane. Its boundary, the unit circle of the complex plane, is denoted by $\partial D$. Let

$$
\mathcal{K}_{n}:=\left\{p_{n}: p_{n}(z)=\sum_{k=0}^{n} a_{k} z^{k}, a_{k} \in \mathbb{C},\left|a_{k}\right|=1\right\} .
$$

The class $\mathcal{K}_{n}$ is often called the collection of all (complex) unimodular polynomials of degree $n$. Given a sequence $\left(\varepsilon_{n}\right)$ of positive numbers tending to 0 , we say that a sequence $\left(P_{n}\right)$ of unimodular polynomials $P_{n} \in \mathcal{K}_{n}$ is $\left(\varepsilon_{n}\right)$-ultraflat if

$$
\left(1-\varepsilon_{n}\right) \sqrt{n+1} \leq\left|P_{n}(z)\right| \leq\left(1+\varepsilon_{n}\right) \sqrt{n+1}, \quad z \in \partial D, \quad n \in \mathbb{N} .
$$

The existence of ultraflat unimodular polynomials seemed very unlikely, in view of a 1957 conjecture of P. Erdös (Problem 22 in [Er1]) asserting that, for all $P_{n} \in \mathcal{K}_{n}$ with $n \geq 1$,

$$
\max _{z \in \partial D}\left|P_{n}(z)\right| \geq(1+\varepsilon) \sqrt{n+1},
$$

where $\varepsilon>0$ is an absolute constant (independent of $n$ ). Yet, combining some probabilistic lemmas from Körner's paper [Kö] with some constuctive methods (Gauss polynomials, etc.), which were completely unrelated to the deterministic part of Körner's paper, Kahane [Ka] proved that there exists a sequence $\left(P_{n}\right)$ with $P_{n} \in \mathcal{K}_{n}$ which is $\left(\varepsilon_{n}\right)$-ultraflat, where $\varepsilon_{n}=O\left(n^{-1 / 17} \sqrt{\log n}\right)$. Thus the Erdős conjecture was disproved for the classes $\mathcal{K}_{n}$.

In this paper we study ultraflat sequences $\left(P_{n}\right)$ of unimodular polynomials $P_{n} \in$ $\mathcal{K}_{n}$ in general, not necessarily those produced by Kahane in his paper [Ka]. We prove a few conjectures of Saffari [Sa] (see also [QS2]). Most importantly the following one.

Uniform Distribution Conjecture for the Angular Speed. Let $\left(P_{n}\right)$ be a $\varepsilon_{n}$ ultraflat sequence of unimodular polynomials $P_{n} \in \mathcal{K}_{n}$. Let

$$
P_{n}\left(e^{i t}\right)=R_{n}(t) e^{i \alpha_{n}(t)}, \quad R_{n}(t)=\left|P_{n}\left(e^{i t}\right)\right| .
$$

In the interval $[0,2 \pi]$, the distribution of the normalized angular speed $\alpha_{n}^{\prime}(t) / n$ converges to the uniform distribution as $n \rightarrow \infty$. More precisely, we have

$$
\mathrm{m}\left\{t \in[0,2 \pi]: 0 \leq \alpha_{n}^{\prime}(t) \leq n x\right\}=2 \pi x+o_{n}(x)
$$

for every $x \in[0,1]$, where $\lim _{n \rightarrow \infty} o_{n}(x)=0$ for every $x \in[0,1]$.

1991 Mathematics Subject Classification. 41A17.

Key words and phrases. unimodular polynomials, ultraflat polynomials, angular derivatives. Research supported in part by the NSF of the USA under Grant No. DMS-9623156. 


\section{INTRODUCTION}

Let $D$ be the open unit disk of the complex plane. Its boundary, the unit circle of the complex plane, is denoted by $\partial D$. Let

$$
\mathcal{K}_{n}:=\left\{p_{n}: p_{n}(z)=\sum_{k=0}^{n} a_{k} z^{k}, a_{k} \in \mathbb{C},\left|a_{k}\right|=1\right\} .
$$

The class $\mathcal{K}_{n}$ is often called the collection of all (complex) unimodular polynomials of degree $n$. Let

$$
\mathcal{L}_{n}:=\left\{p_{n}: p_{n}(z)=\sum_{k=0}^{n} a_{k} z^{k}, a_{k} \in\{-1,1\}\right\} .
$$

The class $\mathcal{L}_{n}$ is often called the collection of all (real) unimodular polynomials of degree $n$. By Parseval's formula,

$$
\int_{0}^{2 \pi}\left|P_{n}\left(e^{i t}\right)\right|^{2} d t=2 \pi(n+1)
$$

for all $P_{n} \in \mathcal{K}_{n}$. Therefore

$$
\min _{z \in \partial D}\left|P_{n}(z)\right|<\sqrt{n+1}<\max _{z \in \partial D}\left|P_{n}(z)\right|
$$

for all $P_{n} \in \mathcal{K}_{n}$.

An old problem (or rather an old theme) is the following.

Problem 1.1 (Littlewood's Flatness Problem). Examine that how close a unimodular polynomial $P_{n} \in \mathcal{K}_{n}$ or $P_{n} \in \mathcal{L}_{n}$ can come to satisfying

$$
\left|P_{n}(z)\right|=\sqrt{n+1}, \quad z \in \partial D .
$$

Obviously (1.2) is impossible if $n \geq 1$. So one must look for less than (1.2), but then there are various ways of seeking such an "approximate situation". One way is the following. In his paper [Li1] Littlewood had suggested that, conceivably, there might exist a sequence $\left(P_{n}\right)$ of polynomials $P_{n} \in \mathcal{K}_{n}$ (possibly even $P_{n} \in \mathcal{L}_{n}$ ) such that $(n+1)^{-1 / 2}\left|P_{n}\left(e^{i t}\right)\right|$ converge to 1 uniformly in $t \in \mathbb{R}$. We shall call such sequences of unimodular polynomials "ultraflat". More precisely, we give the following definitions. In the rest of the paper, we assume that $\left(n_{k}\right)$ is a strictly increasing sequence of positive integers.

Definition 1.2. Given a positive number $\varepsilon$, we say that a polynomial $P_{n} \in \mathcal{K}_{n}$ is $\varepsilon$-flat if

$$
(1-\varepsilon) \sqrt{n+1} \leq\left|P_{n}(z)\right| \leq(1+\varepsilon) \sqrt{n+1}, \quad z \in \partial D,
$$

or equivalently

$$
\max _{z \in \partial D}|| P_{n}(z)|-\sqrt{n+1}| \leq \varepsilon \sqrt{n+1} .
$$


Definition 1.3. Given a sequence $\left(\varepsilon_{n_{k}}\right)$ of positive numbers tending to 0 , we say that a sequence $\left(P_{n_{k}}\right)$ of unimodular polynomials $P_{n_{k}} \in \mathcal{K}_{n_{k}}$ is $\left(\varepsilon_{n_{k}}\right)$-ultraflat if

$$
\left(1-\varepsilon_{n_{k}}\right) \sqrt{n_{k}+1} \leq\left|P_{n_{k}}(z)\right| \leq\left(1+\varepsilon_{n_{k}}\right) \sqrt{n_{k}+1}, \quad z \in \partial D,
$$

or equivalently

$$
\max _{z \in \partial D}|| P_{n_{k}}(z)\left|-\sqrt{n_{k}+1}\right| \leq \varepsilon_{n_{k}} \sqrt{n_{k}+1}
$$

The existence of an ultraflat sequence of unimodular polynomials seemed very unlikely, in view of a 1957 conjecture of P. Erdős (Problem 22 in [Er1]) asserting that, for all $P_{n} \in \mathcal{K}_{n}$ with $n \geq 1$,

$$
\max _{z \in \partial D}\left|P_{n}(z)\right| \geq(1+\varepsilon) \sqrt{n+1}
$$

where $\varepsilon>0$ is an absolute constant (independent of $n$ ). Yet, combining some probabilistic lemmas from Körner's paper [Kö] with some constuctive methods (Gauss polynomials, etc.), which were completely unrelated to the deterministic part of Körner's paper, Kahane [Ka] proved that there exists a sequence $\left(P_{n}\right)$ with $P_{n} \in \mathcal{K}_{n}$ which is $\left(\varepsilon_{n}\right)$-ultraflat, where

$$
\varepsilon_{n}=O\left(n^{-1 / 17} \sqrt{\log n}\right)
$$

Thus the Erdős conjecture (1.5) was disproved for the classes $\mathcal{K}_{n}$. For the more restricted class $\mathcal{L}_{n}$ the analogous Erdös conjecture is unsettled to this date. It is a common belief that the analogous Erdős conjecture for $\mathcal{L}_{n}$ is true, and consequently there is no ultraflat sequence of unimodular polynomials $P_{n} \in \mathcal{L}_{n}$. I thank $\mathrm{H}$. Queffelec for providing more details about the existence of ultraflat sequences $\left(P_{n}\right)$ of unimodular polynomials $P_{n} \in \mathcal{K}_{n}$. The story is roughly the following.

Littlewood [Li1] had constructed polynomials $P_{n} \in \mathcal{K}_{n}$ so that on one hand $\left|P_{n}(z)\right| \leq B \sqrt{n+1}$ for every $z \in \partial D$, and on the other hand $\left|P_{n}(z)\right| \geq A \sqrt{n+1}$ with an absolute constant $A>0$ for every $z \in \partial D$ except for a small arc. In the light of this result he asked how close we can get to satisfying $\left|P_{n}(z)\right|=\sqrt{n+1}$ for every $z \in \partial D$ if $P_{n} \in \mathcal{K}_{n}$. The first result in this direction is due to Körner [Kör]. By using a result of Byrnes, he showed that there are absolute constants $0<A<B$ such that $A \sqrt{n+1} \leq\left|P_{n}(z)\right| \leq B \sqrt{n+1}$ for every $z \in \partial D$. Then Kahane [Ka] constructed a sequence $\left(P_{n}\right)$ of polynomials $P_{n} \in \mathcal{K}_{n}$ for which

$$
\left(1-\varepsilon_{n}\right) \sqrt{n+1} \leq\left|P_{n}(z)\right| \leq\left(1+\varepsilon_{n}\right) \sqrt{n+1}, \quad z \in \partial D,
$$

with a sequence $\left(\varepsilon_{n}\right)$ of positive real numbers converging to 0 . Such a sequence is called $\left(\varepsilon_{n}\right)$-ultraflat.

Kahane's construction seemed to indicate a very rigid behavior for the phase function $\alpha_{n}$, where

$$
P_{n}\left(e^{i t}\right)=R_{n}(t) e^{i \alpha_{n}(t)}, \quad R_{n}(t)=\left|P_{n}\left(e^{i t}\right)\right| .
$$


Saffari [Sa] had conjectured in 1991 that for every ultraflat sequence $\left(P_{n}\right), \alpha_{n}^{\prime}(t) / n$ converges in measure to the uniform distribution on $[0,1]$, that is,

$$
m\left\{t \in[0,2 \pi]: 0 \leq \alpha_{n}^{\prime}(t) \leq n x\right\} \rightarrow 2 \pi x, \quad 0 \leq x \leq 1,
$$

where $m$ is the Lebesgue measure on the Borel subsets of $[0,2 \pi)$. Since it can be seen easily that $X_{n}:=\alpha_{n}^{\prime}(t) / n$ is uniformly bounded, the method of moments applies and everything could be obtained from

$$
\int_{0}^{1} X_{n}^{q}(t) d t=\frac{1}{q+1}, \quad q=0,1, \ldots
$$

This was proved by Saffari [Sa] for $q=0,1,2$. Then in 1996 Queffelec and Saffari [QS2] used Kahane's method with a slight modification to show the existence of an ultraflat sequence $\left(P_{n}\right)$ which satisfies (1.7). They also showed that (1.8) is true for $q=3$ (and almost for $q=4$ ) for any ultraflat sequence $\left(P_{n}\right)$ of polynomials $P_{n} \in \mathcal{K}_{n}$. When their work was submitted to Journal of Fourier Analysis and Applications, the editor in chief, J. Benedetto, and one of his students discovered an error in Byrnes work which, as a result, invalidated Körner's work. It was discovered that the deterministic part of Körner's [Kö] work was incorrect, and it was based on the incorrect "Theorem 2" of Byrnes' paper [By]. For details of the story see the forthcoming paper by by J.S. Byrnes and B. Saffari [BS].

Fortunately Kahane's work was independent of Byrnes'. It contained though an other slight error which was corrected in [QS2]. Ultraflat sequences $\left(P_{n}\right)$ of polynomials $P_{n} \in \mathcal{K}_{n}$ do exist! It is important to note this, otherwise the work of this paper would be without object. In this paper we answer Saffari's Problem affirmatively, namely we show that (1.7) (or equivalently (1.8)) is true for every ultraflat sequence $\left(P_{n}\right)$ of unimodular polynomials $P_{n} \in \mathcal{K}_{n}$.

An interesting related result to Kahane's breakthrough is given in [Be]. For an account of some of the work done till the mid 1960's, see Littlewood's book [Li2] and [QS2].

In this paper we study ultraflat sequences $\left(P_{n}\right)$ of unimodular polynomials $P_{n} \in$ $\mathcal{K}_{n}$ in general, not necessarily those produced by Kahane in his paper [Ka]. With trivial modifications our results remain valid even if we study ultraflat sequences $\left(P_{n_{k}}\right)$ of unimodular polynomials $P_{n_{k}} \in \mathcal{K}_{n_{k}}$. It is left to the reader to formulate these analogue results.

\section{The Phase Problem: Results and Conjectures of Saffari}

Let $\left(\varepsilon_{n}\right)$ be a sequence of positive numbers tending to 0 . So $\varepsilon_{n}<1 / 3$ for all sufficiently large large $n=1,2, \ldots$ The assumption that the sequence $\left(P_{n}\right)$ of unimodular polynomials $P_{n} \in \mathcal{K}_{n}$ is $\left(\varepsilon_{n}\right)$-ultraflat will be denoted by $\left(P_{n}\right) \in$ $\mathrm{UF}\left(\left(\varepsilon_{n}\right)\right)$. Let $\left(P_{n}\right) \in \mathrm{UF}\left(\left(\varepsilon_{n}\right)\right)$. We write

$$
P_{n}\left(e^{i t}\right)=R_{n}(t) e^{i \alpha_{n}(t)}, \quad R_{n}(t)=\left|P_{n}\left(e^{i t}\right)\right| .
$$

It is a simple exercise to show that $\alpha_{n}$ can be chosen to be in $C^{\infty}(\mathbb{R})$. This is going to be our understanding throughout the paper. We think of $t$ as time. The 
ultraflatness condition (1.3) means that the mobile point $P_{n}\left(e^{i t}\right)$ moves inside a narrow annulus centered at the origin and of inner radius $\left(1-\varepsilon_{n}\right) \sqrt{n+1}$ and of outer radius $\left(1+\varepsilon_{n}\right) \sqrt{n+1}$. Our purpose is the phase problem, that is the study of the phase $\alpha_{n}(t)$, or rather the (instantaneous) angular speed $\alpha_{n}^{\prime}(t)$. Writing

$$
P_{n}\left(e^{i t}\right)=\sum_{k=0}^{n} \exp \left(i k t+i \theta_{k}\right), \quad \theta_{k} \in \mathbb{R}, \quad k=0,1, \ldots, n
$$

we see that we have $n+1$ unit vectors whose endpoints $\exp \left(i \theta_{k}\right), \quad k=0,1, \ldots, n$, rotate along the unit circle. That $\left(P_{n}\right) \in \operatorname{UF}\left(\left(\varepsilon_{n}\right)\right)$ is equivalent to saying that there is a choice of initial positions $\exp \left(i \theta_{k}\right)$ so that the resultant vector has endpoint $P_{n}\left(e^{i t}\right)$ moving in the above mentioned narrow annulus. Our intuition may tell us two things. First that, since the "components" $\exp (i k t)$ have (respective) angular speeds $0,1,2 \ldots, n$, then the "resultant angular speed is" is approximately their average; in other words, we might expect to have

$$
\alpha_{n}^{\prime}(t)=n / 2+o_{n}(t) n, \quad \lim _{n \rightarrow \infty} \max _{0 \leq t \leq 2 \pi} o_{n}(t)=0, \quad t \in[0,2 \pi] .
$$

However, Saffari observed that this is true in average, that is

$$
\frac{1}{2 \pi} \int_{0}^{2 \pi} \alpha_{n}^{\prime}(t) d t=n / 2+o_{n} n, \quad \lim _{n \rightarrow \infty} o_{n}=0
$$

but that (2.2) itself is far from being true. He proves that $\alpha_{n}^{\prime}(t)$ takes values at least as large as $2 n / 3+o_{n} n$ and as small as $n / 3+o_{n} n$ with suitable constants $o_{n}$ and $o_{n}^{*}$ converging to 0 . Secondly, one may suspect that, since all the components $\exp \left(i k t+i \theta_{k}\right)$ turn counter-clockwise, then so does their resultant $P_{n}\left(e^{i t}\right)$, modulo negligible fluctuations: in other words,

$$
\min _{0 \leq t \leq 2 \pi} \alpha_{n}^{\prime}(t) \geq o_{n} n
$$

with suitable constants $o_{n}$ converging to 0 . Saffari [Sa] proves that this is indeed true, moreover

$$
o_{n} n \leq \alpha_{n}^{\prime}(t) \leq n-o_{n} n
$$

with suitable constants $o_{n}$ converging to 0 . He conjectures the following. Let $\left(P_{n}\right) \in \operatorname{UF}\left(\left(\varepsilon_{n}\right)\right)$. Then, with the notation $(2.1)$, we have

$$
\min _{0 \leq t \leq 2 \pi} \alpha_{n}^{\prime}(t)=o_{n} n \quad \text { and } \quad \max _{0 \leq t \leq 2 \pi} \alpha_{n}^{\prime}(t)=n+o_{n}^{*} n
$$

with suitable constants $o_{n}$ and $o_{n}^{*}$ converging to 0 .

In Section 4 we prove this conjecture. In fact, Saffari [Sa] conjectures something much more specific: 
Conjecture 2.1 (Uniform Distribution Conjecture for the Angular Speed). Suppose $\left(P_{n}\right) \in \operatorname{UF}\left(\left(\varepsilon_{n}\right)\right)$. Then, with the notation (2.1), in the interval $[0,2 \pi]$, the distribution of the normalized angular speed $\alpha_{n}^{\prime}(t) / n$ converges to the uniform distribution as $n \rightarrow \infty$. More precisely, we have

$$
\mathrm{m}\left\{t \in[0,2 \pi]: 0 \leq \alpha_{n}^{\prime}(t) \leq n x\right\}=2 \pi x+o_{n}(x)
$$

for every $x \in[0,1]$, where $\lim _{n \rightarrow \infty} o_{n}(x)=0$ for every $x \in[0,1]$, As a consequence, the distribution of $\left|P_{n}^{\prime}\left(e^{i t}\right)\right| / n^{3 / 2}$ also converges to the uniform distribution as $n \rightarrow$ $\infty$. More precisely, we have

$$
\mathrm{m}\left\{t \in[0,2 \pi]: 0 \leq\left|P_{n}^{\prime}\left(e^{i t}\right)\right| \leq n^{3 / 2} x\right\}=2 \pi x+o_{n}(x)
$$

for every $x \in[0,1]$, where $\lim _{n \rightarrow \infty} o_{n}(x)=0$ for every $x \in[0,1]$.

In both statements the convergence of $o_{n}(x)$ is uniform on $[0,1]$ by Dini's Theorem.

The basis of this conjecture was that for the special ultraflat sequences of unimodular polynomials produced by Kahane [Ka], (2.7) is indeed true. In Section 4 we prove this conjecture in general.

In the general case (2.7) can, by integration, be reformulated (equivalently) in terms of the moments of the angular speed $\alpha_{n}^{\prime}(t)$. This was observed and recorded by Saffari [Sa]. For completeness we will present the proof of this equivalence in Section 4 and we will settle Conjecture 2.1 by proving the following result.

Theorem 2.2 (Reformulation of the Uniform Distribution Conjecture). Let $\left(P_{n}\right) \in \mathrm{UF}\left(\left(\varepsilon_{n}\right)\right)$. Then, for any $q>0$ we have

$$
\frac{1}{2 \pi} \int_{0}^{2 \pi}\left|\alpha_{n}^{\prime}(t)\right|^{q} d t=\frac{n^{q}}{q+1}+o_{n, q} n^{q} .
$$

with suitable constants $o_{n, q}$ converging to 0 as $n \rightarrow \infty$ for every fixed $q>0$.

An immediate consequence of (2.9) is the remarkable fact that for large values of $n \in \mathbb{N}$, the $L_{q}(\partial D)$ Bernstein factors

$$
\frac{\int_{0}^{2 \pi}\left|P_{n}^{\prime}\left(e^{i t}\right)\right|^{q} d t}{\int_{0}^{2 \pi}\left|P_{n}\left(e^{i t}\right)\right|^{q} d t}
$$

of the elements of ultraflat sequences $\left(P_{n}\right)$ of unimodular polynomials are essentially independent of the polynomials. More precisely (2.9) implies the following result.

Theorem 2.3 (The Bernstein Factors). Let $q$ be an arbitrary positive real number. Let $\left(P_{n}\right) \in \operatorname{UF}\left(\left(\varepsilon_{n}\right)\right)$. We have

$$
\frac{\int_{0}^{2 \pi}\left|P_{n}^{\prime}\left(e^{i t}\right)\right|^{q} d t}{\int_{0}^{2 \pi}\left|P_{n}\left(e^{i t}\right)\right|^{q} d t}=\frac{n^{q+1}}{q+1}+o_{n, q} n^{q+1},
$$


and as a limit case,

$$
\frac{\max _{0 \leq t \leq 2 \pi}\left|P_{n}^{\prime}\left(e^{i t}\right)\right|}{\max _{0 \leq t \leq 2 \pi}\left|P_{n}\left(e^{i t}\right)\right|}=n+o_{n} n .
$$

with suitable constants $o_{n, q}$ and $o_{n}$ converging to 0 as $n \rightarrow \infty$ for every fixed $q$.

In Section 3 we will show the following result which turns out to be stronger than Theorem 2.2.

Theorem 2.4 (Negligibility Theorem for Higher Derivatives). Let $\left(P_{n}\right) \in$ $\mathrm{UF}\left(\left(\varepsilon_{n}\right)\right)$. For every integer $r \geq 2$, we have

$$
\max _{0 \leq t \leq 2 \pi}\left|\alpha_{n}^{(r)}(t)\right| \leq o_{n, r} n^{r}
$$

with suitable constants $o_{n, r}>0$ converging to 0 for every fixed $r=2,3, \ldots$

We will show in Section 4 how Theorem 2.1 follows from Theorem 2.4.

Finally we give an extension of Saffari's Uniform Distribution Conjecture to higher derivatives. This will be shown in Section 4 .

Theorem 2.5 (Distribution of the Modulus of Higher Derivatives of Ultraflat Sequences of Unimodular Polynomials). Suppose $\left(P_{n}\right) \in \mathrm{UF}\left(\left(\varepsilon_{n}\right)\right)$. Then

$$
\left(\frac{\left|P_{n}^{(r)}\left(e^{i t}\right)\right|}{n^{r+1 / 2}}\right)^{1 / r}
$$

converges to the uniform distribution as $n \rightarrow \infty$. More precisely, we have

$$
\mathrm{m}\left\{t \in[0,2 \pi]: 0 \leq\left|P_{n}^{(r)}\left(e^{i t}\right)\right| \leq n^{r+1 / 2} x^{r}\right\}=2 \pi x+o_{r, n}(x)
$$

for every $x \in[0,1]$, where $\lim _{n \rightarrow \infty} o_{r, n}(x)=0$ for every fixed $r=1,2, \ldots$ and $x \in[0,1]$.

For every fixed $r=1,2, \ldots$, the convergence of $o_{n, r}(x)$ is uniform on $[0,1]$ by Dini's Theorem.

\section{Proof of Theorem 2.4}

To prove Theorem 2.4 we need a few lemmas. The first one is a standard polynomial inequality ascribed to Bernstein. Its proof is a simple exercise in complex analysis (an application of the Maximum Principle), and it may be found in a number of books. See [BE, p. 390], for example. We will use the more or less standard notation

$D\left(z_{0}, R\right):=\left\{z \in \mathbb{C}:\left|z-z_{0}\right|<R\right\}, \quad$ and $\quad \bar{D}\left(z_{0}, r\right):=\left\{z \in \mathbb{C}:\left|z-z_{0}\right| \leq R\right\}$. 
Lemma 3.1. We have

$$
|p(z)| \leq|z|^{n} \max _{u \in \bar{D}(0,1)}|p(u)|
$$

for every polynomial $p$ of degree at most $n$ with complex coefficients, and for every $z \in \mathbb{C}$ with $|z|>1$. As a corollary (consider $e^{\text {int }} T_{n}(t)$ ), if

$$
T_{n}(t)=\sum_{k=-n}^{n} c_{k} e^{i k t}, \quad c_{k} \in \mathbb{C}
$$

satisfies $\left|T_{n}(t)\right| \leq M$ for all $t \in \mathbb{R}$, then it satisfies $\left|T_{n}(t)\right| \leq M e^{n \operatorname{Im}(t)}$ for all $t \in \mathbb{C}$.

The main tool to prove Theorem 2.4 is the following well-known Jensen's Formula. For its proof, see, for example, E.10 c] of Section 4.2 in [BE].

Lemma 3.2 (Jensen's Formula). Suppose $h$ is a nonnegative integer and

$$
f(z)=\sum_{k=h}^{\infty} c_{k} z^{k}, \quad c_{h} \neq 0
$$

is analytic on a disk $D\left(0, R^{\prime}\right)$ with some $R^{\prime}>R$. Suppose that the zeros of $f$ in $D(0, R) \backslash\{0\}$ are $a_{1}, a_{2}, \ldots, a_{m}$, where each zero is listed as many times as its multiplicity. Then

$$
\log \left|c_{h}\right|+h \log R+\sum_{k=1}^{m} \log \frac{R}{\left|a_{k}\right|}=\frac{1}{2 \pi} \int_{0}^{2 \pi} \log \left|f\left(R e^{i \theta}\right)\right| d \theta .
$$

Lemma 3.3. Suppose $\left(\varepsilon_{n}\right)$ is a sequence of numbers from $(0,1 / 3)$ tending to 0 . Suppose $\left(P_{n}\right) \in \mathrm{UF}\left(\left(\varepsilon_{n}\right)\right)$. Then $P_{n}$ does not have a zero in the open annulus

$$
\left\{z \in \mathbb{C}: 1-\frac{1}{2 n \delta_{n}}<|z|<1+\frac{1}{2 n \delta_{n}}\right\}
$$

where the positive numbers $\delta_{n}=\max \left\{2 / \log \left(1 /\left(3 \varepsilon_{n}\right)\right), 1 / n\right\}$ tend to 0 .

Proof of Lemma 3.3. Associated with a polynomial

$$
p_{n}(z)=\sum_{j=0}^{n} a_{j} z^{j}, \quad a_{j} \in \mathbb{C},
$$

we define

$$
p_{n}^{*}(z)=z^{n} \overline{p_{n}(1 / \bar{z})}=\sum_{j=0}^{n} \bar{a}_{n-j} z^{j} .
$$

Let $\left(P_{n}\right) \in \mathrm{UF}\left(\left(\varepsilon_{n}\right)\right)$, that is, $P_{n} \in \mathcal{K}_{n}$ satisfies

$$
\left(1-\varepsilon_{n}\right) \sqrt{n+1}<\left|P_{n}(z)\right|<\left(1+\varepsilon_{n}\right) \sqrt{n+1}
$$


for every $z \in \mathbb{C}$ with $|z|=1$. In fact, in this proof we will not use that $P_{n} \in \mathcal{K}_{n}$, we will use only that $P_{n}$ is a polynomial of degree $n$ with complex coefficients that satisfies

$$
\left(1-\varepsilon_{n}\right) \sqrt{n+1} \leq\left|P_{n}(z)\right| \leq\left(1+\varepsilon_{n}\right) \sqrt{n+1}, \quad z \in \partial D .
$$

Then

$$
\left(1-\varepsilon_{n}\right)^{2}(n+1)<z^{-n} P_{n}(z) P_{n}^{*}(z)=\left|P_{n}(z)\right|^{2}<\left(1+\varepsilon_{n}\right)^{2}(n+1)
$$

for every $z \in \partial D$. We define

$$
Q_{n}(z)=P_{n}(z) P_{n}^{*}(z)-(n+1) z^{n} .
$$

Then $Q_{n}$ is a polynomial of degree $2 n$ and

$$
-3 \varepsilon_{n}(n+1)<z^{-n} Q_{n}(z)=\left|P_{n}(z)\right|^{2}-(n+1)<3 \varepsilon_{n}(n+1)
$$

for every $z \in \partial D$. From this we conclude that

$$
\left|Q_{n}(z)\right|<3 \varepsilon_{n}(n+1)
$$

for every $z \in \mathbb{C}$ with $|z|=1$. Using Lemma 3.1 and (3.3), we obtain that

$$
\left|Q_{n}(z)\right| \leq|z|^{2 n} 3 \varepsilon_{n}(n+1)<n+1
$$

for every $z \in \mathbb{C}$ for which

$$
1 \leq|z|<1+\frac{1}{n \delta_{n}}
$$

with $\delta_{n}=\max \left\{2 / \log \left(1 /\left(3 \varepsilon_{n}\right)\right), 1 / n\right\}$. Suppose that $P_{n}$ has a zero in the annulus

$$
\left\{z \in \mathbb{C}: 1-\frac{1}{2 n \delta_{n}}<|z|<1+\frac{1}{2 n \delta_{n}}\right\} .
$$

Then $P_{n} P_{n}^{*}$ has a zero $z_{0}$ in the annulus

$$
\left\{z \in \mathbb{C}: 1 \leq|z|<1+\frac{1}{n \delta_{n}}\right\} .
$$

Hence by (3.2) we have

$$
\left|Q_{n}\left(z_{0}\right)\right|=\left|P_{n}\left(z_{0}\right) P_{n}^{*}\left(z_{0}\right)-(n+1) z_{0}^{n}\right|=(n+1)\left|z_{0}\right|^{n} \geq n+1,
$$

which is impossible by (3.4).

Lemma 3.4. Suppose $\left(\varepsilon_{n}\right)$ is a sequence of numbers from $(0,1 / 3)$ tending to 0 . Suppose $\left(P_{n}\right) \in \operatorname{UF}\left(\left(\varepsilon_{n}\right)\right)$. Let $1 / n<R<2$. Let $z_{0} \in \partial D$. Then $P_{n}$ has at most $5 n R$ zeros in the disk $D\left(z_{0}, R\right)$.

Proof. We use Jensen's formula on the disk $D\left(z_{0}, 2 R\right)$. Note that $\left(P_{n}\right) \in \mathrm{UF}\left(\left(\varepsilon_{n}\right)\right)$ implies

$$
\log \left|P_{n}\left(z_{0}\right)\right| \geq \frac{1}{2} \log (n+1)+\log \left(1-\varepsilon_{n}\right) \geq \frac{1}{2} \log (n+1)-\frac{1}{2},
$$


while on the boundary of the disk $D\left(z_{0}, 2 R\right)$ one can estimate $\left|P_{n}(z)\right|$ by the Bernstein inequality given by Lemma 3.1:

$$
\left|P_{n}(z)\right| \leq\left(1+\varepsilon_{n}\right) \sqrt{n+1}(1+2 R)^{n},
$$

that is,

$$
\log \left|P_{n}(z)\right| \leq \frac{1}{2} \log (n+1)+\frac{1}{3}+n(2 R) .
$$

on the boundary of $D\left(z_{0}, 2 R\right)$. Now if $m$ denotes the number of zeros of $P_{n}$ in $D\left(z_{0}, R\right)$, then by Jensen's formula

$$
\frac{1}{2} \log (n+1)-\frac{1}{2}+m \log 2 \leq \frac{1}{2} \log (n+1)+\frac{1}{3}+2 n R,
$$

whence

$$
m \leq \frac{3 n R}{\log 2} \leq 5 n R
$$

and the lemma is proved.

Our last lemma is a well-known inequality in approximation theory.

Lemma 3.5 (Bernstein's Inequality). If $P_{n}$ is a polynomial of degree at most $n$ with complex coefficients, then

$$
\max _{z \in \partial D}\left|P_{n}^{\prime}(z)\right| \leq n \max _{z \in \partial D}\left|P_{n}(z)\right| .
$$

Now we are ready for the proof of Theorem 2.4.

Proof of Theorem 2.4. It is easy to find a formula for $\alpha_{n}(t)$ in terms of $P_{n}$. One can easily verify formula (8.2) from Saffari's paper [Sa], which asserts that

$$
\alpha_{n}^{\prime}(t)=\operatorname{Re}\left(\frac{e^{i t} P_{n}^{\prime}\left(e^{i t}\right)}{P_{n}\left(e^{i t}\right)}\right) .
$$

Observe that if $z_{1}, z_{2}, \ldots, z_{n}$ denote the zeros of $P_{n}$ in the complex plane, then

$$
\frac{z P_{n}^{\prime}(z)}{P_{n}(z)}=\sum_{j=1}^{n} \frac{z}{z-z_{j}}=\sum_{j=1}^{n}\left(1+\frac{z_{j}}{z-z_{j}}\right) .
$$

Since $P_{n}$ is unimodular, its zeros satisfy

$$
1 / 2 \leq\left|z_{1}\right|,\left|z_{2}\right|, \ldots,\left|z_{n}\right| \leq 2 .
$$

To see the upper bound, for example, let

$$
P_{n}(z)=\sum_{j=0}^{n} a_{j} z^{j}, \quad a_{j} \in \mathbb{C}, \quad\left|a_{j}\right|=1 .
$$


Now if $z_{0} \in \mathbb{C}$ and $\left|z_{0}\right|>2$, then

$$
\left|\sum_{j=0}^{n} a_{j} z_{0}^{j}\right| \geq\left|z_{0}\right|^{n}-\left(\left|z_{0}\right|^{n-1}+\left|z_{0}\right|^{n-2}+\cdots+\left|z_{0}\right|^{1}+\left|z_{0}\right|^{0}\right)=\left|z_{0}\right|^{n}-\frac{\left|z_{0}\right|^{n}-1}{\left|z_{0}\right|-1}>0 .
$$

Using (3.5) and (3.6) and substituting $z_{0}=e^{i t_{0}}$, we can give the following upper bound (the constants $A_{m}$ below depend only on $m$ ):

$$
\begin{aligned}
\left|\alpha_{n}^{(r)}\left(t_{0}\right)\right| & =\left|\frac{d^{r-1}}{d t^{r-1}}\left(\operatorname{Re}\left(\frac{e^{i t} P_{n}^{\prime}\left(e^{i t}\right)}{P_{n}\left(e^{i t}\right)}\right)\right)\left(t_{0}\right)\right| \leq\left|\frac{d^{r-1}}{d t^{r-1}}\left(\frac{e^{i t} P_{n}^{\prime}\left(e^{i t}\right)}{P_{n}\left(e^{i t}\right)}\right)\left(t_{0}\right)\right| \\
& =\left|\sum_{m=0}^{r-1} A_{m} \frac{d^{m}}{d z^{m}}\left(\frac{z P_{n}^{\prime}(z)}{P_{n}(z)}\right)\left(z_{0}\right) e^{i m t_{0}}\right| \\
& =\left|\sum_{m=0}^{r} A_{m} \frac{d^{m}}{d z^{m}}\left(\sum_{k=1}^{n}\left(1+\frac{z_{k}}{z-z_{k}}\right)\right)\left(z_{0}\right) e^{i m t_{0}}\right| \\
& \leq\left|A_{0} \frac{z_{0} P_{n}^{\prime}\left(z_{0}\right)}{P_{n}\left(z_{0}\right)}\right|+\sum_{m=1}^{r-1}\left|A_{m}\right| m ! \sum_{k=1}^{n}\left|z_{k}\right|\left|z_{0}-z_{k}\right|^{-(m+1)} \\
& \leq\left|A_{0} \frac{z_{0} P_{n}^{\prime}\left(z_{0}\right)}{P_{n}\left(z_{0}\right)}\right|+2 \sum_{m=1}^{r-1}\left|A_{m}\right| m ! \sum_{k=1}^{n}\left|z_{0}-z_{k}\right|^{-(m+1)} .
\end{aligned}
$$

Now we define the annulus

$$
E_{\mu}=D\left(z_{0}, \frac{2^{\mu}}{2 n \delta_{n}}\right) \backslash D\left(z_{0}, \frac{2^{\mu-1}}{2 n \delta_{n}}\right), \quad \mu=1,2, \ldots,
$$

where $\delta_{n}:=\max \left\{2 / \log \left(1 /\left(3 \varepsilon_{n}\right)\right), 1 / n\right\}$ as in Lemma 3.3. We denote the number of zeros of $P_{n}$ in $E_{\mu}$ by $m_{\mu}$. By Lemma $3.4 m_{\mu} \leq 5 n 2^{\mu} /\left(2 n \delta_{n}\right)$. Combining this with (3.7) and Lemmas 3.5 and 3.3, we obtain

$$
\begin{aligned}
\left|\alpha_{n}^{(r)}(t)\right| & \leq C_{0} \frac{n\left(1+\varepsilon_{n}\right) \sqrt{n}}{\left(1-\varepsilon_{n}\right) \sqrt{n}}+C_{r} \sum_{m=1}^{r-1} \sum_{k=1}^{n}\left|z_{0}-z_{k}\right|^{-(m+1)} \\
& \leq 2 C_{0} n+C_{r} \sum_{m=1}^{r-1} \sum_{\mu=1}^{\infty} m_{\mu}\left(\frac{2^{\mu-1}}{2 n \delta_{n}}\right)^{-(m+1)} \\
& \leq 2 C_{0} n+C_{r} \sum_{m=1}^{r-1} \sum_{\mu=1}^{\infty} \frac{5 n 2^{\mu}}{2 n \delta_{n}}\left(\frac{2^{\mu-1}}{2 n \delta_{n}}\right)^{-(m+1)} \\
& \leq 2 C_{0} n+C_{r} \sum_{m=1}^{r-1} \sum_{\mu=1}^{\infty} 2 \cdot 2^{-(\mu-1) m} 5 n\left(2 n \delta_{n}\right)^{m} \\
& \leq 2 C_{0} n+C_{r}^{\prime} n^{r} \delta_{n} \leq C_{r}^{\prime \prime} n^{r} \delta_{n}
\end{aligned}
$$

where $C_{r}, C_{r}^{\prime}$, and $C_{r}^{\prime \prime}$ are positive constants depending only on $r$. Since

$$
\delta_{n}:=\max \left\{2 / \log \left(1 /\left(3 \varepsilon_{n}\right)\right), 1 / n\right\}
$$

tends to 0 together with $\varepsilon_{n}>0$, the theorem is proved. 


\section{Proof of Conjecture 2.1, Theorem 2.2 And Theorem 2.3}

Our first tool is a classical polynomial inequality of Bernstein available in many books. See [BE, Corollary 5.1.5], for example.

Lemma 4.1 (Bernstein's Inequality for Trigonometric Polynomials). We have

$$
\max _{0 \leq t \leq 2 \pi}\left|T^{(m)}(t)\right| \leq n^{m} \max _{0 \leq t \leq 2 \pi}|T(t)|, \quad m=1,2, \ldots,
$$

for every trigonometric polynomial $T$ of degree at most $n$ with complex coefficients.

First we prove Theorem 2.2 for positive integers $q$. We need the following lemma:

Lemma 4.2. Suppose $\left(\varepsilon_{n}\right)$ is a sequence of numbers from $(0,1 / 3)$ tending to 0 . Suppose $\left(P_{n}\right) \in \mathrm{UF}\left(\left(\varepsilon_{n}\right)\right)$. Using notation (2.1), we have

$$
\max _{0 \leq t \leq 2 \pi}\left|R_{n}^{(m)}(t)\right|=o_{n, m} n^{m+1 / 2}, \quad m=1,2, \ldots,
$$

with suitable constants $o_{n, m}$ converging to 0 as $n \rightarrow \infty$ for every $m=1,2, \ldots$.

Proof of Lemma 4.2. Let $\delta_{n}:=\max \left\{2 / \log \left(1 /\left(3 \varepsilon_{n}\right)\right), 1 / n\right\}$, as in the proof Lemma 3.3. Let $\left(P_{n}\right) \in \mathrm{UF}\left(\left(\varepsilon_{n}\right)\right)$, that is, $P_{n} \in \mathcal{K}_{n}$ satisfies

$$
\left(1-\varepsilon_{n}\right) \sqrt{n+1}<\left|P_{n}(z)\right|<\left(1+\varepsilon_{n}\right) \sqrt{n+1}, \quad z \in \partial D .
$$

(In fact, in this proof we will not use that $P_{n} \in \mathcal{K}_{n}$, we will use only that $P_{n}$ is a polynomial of degree $n$ with complex coefficients that satisfies (4.2).) We will use the $p_{n}^{*}$ notation introduced by (3.1).

Step 1. By Lemma 3.3,

$$
T_{n}(t):=e^{-i n t} P_{n}\left(e^{i t}\right) P_{n}^{*}\left(e^{i t}\right)
$$

has no zeros in the strip

$$
\mathcal{E}_{n}:=\left\{z \in \mathbb{C}:|\operatorname{Im}(z)| \leq \frac{1}{4 n \delta_{n}}\right\} .
$$

Therefore

$$
\widetilde{T}_{n}(t):=\sqrt{e^{-i n t} P_{n}\left(e^{i t}\right) P_{n}^{*}\left(e^{i t}\right)}
$$

is a well-defined analytic function in in the strip $\mathcal{E}_{n}$.

Step 2. We show that

$$
\left|\widetilde{T}_{n}^{\prime}(t)\right| \leq o_{n} n^{3 / 2}, \quad t \in \mathbb{R},
$$

with suitable constants $o_{n}$ converging to 0 . Indeed, $T_{n}$ is a trigonometric polynomial of degree $n$ (with complex coefficients). Note that (4.2) implies that

$$
-3 \varepsilon_{n}(n+1)<T_{n}(t)-(n+1)<3 \varepsilon_{n}(n+1) .
$$


Combining this with Lemma 4.1 (Bernstein's Inequality for Trigonometric Polynomials), we obtain

$$
\begin{aligned}
\max _{0 \leq t \leq 2 \pi}\left|T_{n}^{\prime}(t)\right| & =\max _{0 \leq t \leq 2 \pi}\left|\frac{d}{d t}\left(T_{n}(t)-(n+1)\right)\right| \\
& \leq n \max _{0 \leq t \leq 2 \pi}\left|T_{n}(t)-(n+1)\right| \leq n 3 \varepsilon_{n}(n+1) \\
& \leq 6 \varepsilon_{n} n^{2} .
\end{aligned}
$$

Now

$$
\begin{aligned}
\left|\widetilde{T}_{n}^{\prime}(t)\right| & =\left|\frac{T_{n}^{\prime}(t)}{2 \sqrt{T_{n}(t)}}\right| \leq \frac{6 \varepsilon_{n} n^{2}}{\left(1-\varepsilon_{n}\right) \sqrt{n+1}} \leq \frac{6 \varepsilon_{n}}{\left(1-\varepsilon_{n}\right)} n^{3 / 2} \\
& \leq 9 \varepsilon_{n} n^{3 / 2}=o_{n} n^{3 / 2}, \quad t \in \mathbb{R},
\end{aligned}
$$

with suitable constants $o_{n}$ converging to 0 .

Step 3. Let

$$
\mathcal{F}_{c n}:=\left\{z \in \mathbb{C}:|\operatorname{Im}(z)| \leq \frac{c}{n}\right\} .
$$

We show that there is a sufficiently small absolute constant $c>0$ such that

$$
\left|\widetilde{T}_{n}^{\prime}(t)\right| \leq o_{n} n^{3 / 2}, \quad t \in \mathcal{F}_{c n},
$$

with suitable constants $o_{n}$ converging to 0 . To see this, first note that

$$
\left|\widetilde{T}_{n}^{\prime}(t)\right|=\left|\frac{T_{n}^{\prime}(t)}{2 \sqrt{T_{n}(t)}}\right|,
$$

where $T_{n}$ is defined by (4.3). Using (4.6) and Lemma 3.1 we obtain that

$$
\left|T_{n}^{\prime}(t)\right| \leq o_{n}^{\prime} n^{2} e^{n(c / n)}=o_{n} n^{2}, \quad t \in \mathcal{F}_{c n},
$$

and similarly (4.5) and Lemma 3.1 give

$$
\left|T_{n}(t)\right| \geq n / 2, \quad t \in \mathcal{F}_{c n},
$$

for a sufficiently small absolute constant $c>0$, with suitable constants $o_{n}^{\prime}$ and $o_{n}$ converging to 0 .

Now (4.9) - (4.11) imply that

$$
\left|\widetilde{T}_{n}^{\prime}(t)\right| \leq o_{n} n^{3 / 2}, \quad t \in \mathcal{F}_{c n},
$$

for a sufficiently small absolute constant $c>0$, with suitable constants $o_{n}$ converging to 0 .

Step 4. From Step 3 we conclude by the Cauchy Integral Formula that

$$
\begin{aligned}
\left|\widetilde{T}_{n}^{(m)}(t)\right| & =(m-1) !\left|\int_{\partial D(t, c / n)} \frac{T_{n}^{\prime}(\xi) d \xi}{(\xi-t)^{m}}\right| \\
& \leq \frac{2 \pi c}{n}(m-1) ! o_{n, 1} n^{3 / 2}\left(\frac{c}{n}\right)^{-m}=o_{n, m} n^{m+1 / 2}
\end{aligned}
$$


with suitable constants $o_{n, m}$ converging to 0 as $n \rightarrow \infty$ for every fixed $m=1,2, \ldots$.

Step 5. Note that for $t \in \mathbb{R}$ we have

$$
R_{n}(t)=\left|P_{n}\left(e^{i t}\right)\right|=\sqrt{e^{-i n t} P_{n}\left(e^{i t}\right) P_{n}^{*}\left(e^{i t}\right)}=\widetilde{T}_{n}(t),
$$

hence by Step $4 \max _{0 \leq t \leq 1}\left|R_{n}^{(m)}(t)\right|=o_{n, m} n^{m+1 / 2}$ with suitable constants $o_{n, m}$ converging to 0 as $n \rightarrow \infty$ for every fixed $m=1,2, \ldots$ This proves the lemma.

Now we are ready to prove Theorem 2.2 for positive integers $q$.

Proof of Theorem 2.2 for integers $q \geq 0$. Let $\left(P_{n}\right) \in \mathrm{UF}\left(\left(\varepsilon_{n}\right)\right)$. Using our standard notation introduced by (2.1), we introduce

$$
S_{n}(t):=P_{n}\left(e^{i t}\right)=\sum_{k=0}^{n} a_{k, n} e^{i k t}, \quad\left|a_{k, n}\right|=1
$$

We calculate

$$
\frac{1}{2 \pi} \int_{0}^{2 \pi} S_{n}^{(q)}(t) \overline{S_{n}(t)} d t
$$

in two different ways. On one hand, using orthogonality, we have

$$
\frac{1}{2 \pi} \int_{0}^{2 \pi} S_{n}^{(q)}(t) \overline{S_{n}(t)} d t=i^{q} \sum_{k=0}^{n} k^{q}\left|a_{k, n}\right|^{2}=i^{q} \frac{n^{q+1}}{q+1}+o_{n, q} n^{q+1},
$$

with suitable constants $o_{n, q}$ converging to 0 as $n \rightarrow \infty$ for every fixed $q=0,1, \ldots$

On the other hand, Theorem 2.4, Lemma 4.2, and (2.5) give

$$
\begin{aligned}
S_{n}^{(q)}(t) & =\sum_{k=0}^{q}\left(\begin{array}{l}
q \\
k
\end{array}\right) \frac{d^{k}}{d t^{k}}\left(e^{i \alpha_{n}(t)}\right) R_{n}^{(q-k)}(t) \\
& =\frac{d^{q}}{d t^{q}}\left(e^{i \alpha_{n}(t)}\right) R_{n}(t)+\sum_{k=0}^{q-1}\left(\begin{array}{l}
q \\
k
\end{array}\right) \frac{d^{k}}{d t^{k}}\left(e^{i \alpha_{n}(t)}\right) R_{n}^{(q-k)}(t) \\
& =\frac{d^{q}}{d t^{q}}\left(e^{i \alpha_{n}(t)}\right) R_{n}(t)+\sum_{k=0}^{q-1}\left(\begin{array}{l}
q \\
k
\end{array}\right) c_{n, k}(t) n^{k} o_{n, q-k}(t) n^{q-k+1 / 2} \\
& =\left(e^{i \alpha_{n}(t)} \alpha_{n}^{\prime}(t)^{q} i^{q}+o_{n, q}^{\prime}(t) n^{q}\right) R_{n}(t)+o_{n, q}^{\prime \prime}(t) n^{q+1 / 2}
\end{aligned}
$$

with suitable constants $o_{n, q-k}(t), c_{n, k}(t), o_{n, q}^{\prime}(t)$, and $o_{n, q}^{\prime \prime}(t)$, where

$$
\max _{0 \leq t \leq 2 \pi}\left|o_{n, q-k}(t)\right|
$$

converge to 0 for every fixed $q$ and $k=0,1, \ldots, q-1$,

$$
\max _{0 \leq t \leq 2 \pi}\left|c_{n, k}(t)\right|
$$


is bounded by a constant independent of $n$ for every fixed $k=0,1, \ldots, q-1$, and

$$
\max _{0 \leq t \leq 2 \pi}\left|o_{n, q}^{\prime}(t)\right| \quad \text { and } \quad \max _{0 \leq t \leq 2 \pi}\left|o_{n, q}^{\prime \prime}(t)\right|
$$

converge to 0 as $n \rightarrow \infty$ for every fixed $q$. Now (4.13), (4.14), and (4.16) yield

$$
\begin{gathered}
\frac{1}{2 \pi} \int_{0}^{2 \pi} S_{n}^{(q)}(t) \overline{S_{n}(t)} d t \\
=\frac{1}{2 \pi} \int_{0}^{2 \pi}\left(\left(e^{i \alpha_{n}(t)} \alpha_{n}^{\prime}(t)^{q} i^{q}+o_{n, q}^{\prime}(t) n^{q}\right) R_{n}(t)+o_{n, q}^{\prime \prime}(t) n^{q+1 / 2}\right) R_{n}(t) e^{-i \alpha_{n}(t)} d t \\
=\frac{1}{2 \pi} \int_{0}^{2 \pi}\left(\left(n-o_{n}(t) n\right)\left(\alpha_{n}^{\prime}(t)^{q} i^{q}+o_{n, q}^{\prime}(t) n^{q}\right)+o_{n, q}^{\prime \prime \prime}(t) n^{q+1 / 2+1 / 2}\right) d t \\
=\frac{1}{2 \pi} \int_{0}^{2 \pi} i^{q}\left(n-o_{n, q}(t) n\right) \alpha_{n}^{\prime}(t)^{q} d t+o_{n, q}^{*} n^{q+1},
\end{gathered}
$$

with suitable constants $o_{n, q}^{\prime}(t), o_{n, q}^{\prime \prime}(t), o_{n, q}^{\prime \prime \prime}(t)$, and $o_{n, q}^{*}$, where

$$
\max _{0 \leq t \leq 2 \pi}\left|o_{n, q}^{\prime}(t)\right|, \quad \max _{0 \leq t \leq 2 \pi}\left|o_{n, q}^{\prime \prime}(t)\right|, \quad \max _{0 \leq t \leq 2 \pi}\left|o_{n, q}^{\prime \prime \prime}(t)\right|
$$

and $o_{n, q}^{*}$ converge to 0 as $n \rightarrow \infty$ for every fixed $q$.

Now (4.15) and (4.17) give the statement of the theorem for integers $q \geq 0$.

Proof of Conjecture 2.1. We introduce the normalized distribution functions

$$
F_{n}(x):=\mathrm{m}\left\{t \in[0,2 \pi]: 0 \leq \alpha_{n}^{\prime}(t) \leq n x\right\}, \quad x \in[0,1] .
$$

Each $F_{n}$ is continuous and nondecreasing on $[0,1]$, and

$$
0 \leq F_{n}(x) \leq 2 \pi, \quad x \in[0,1] .
$$

Suppose that the conjecture is not true. Then we can find a subsequence $\left(F_{n_{k}}\right)$ of the sequence $\left(F_{n}\right)$, and numbers $y \in[0,1]$ and $\varepsilon>0$ such that

$$
\left|F_{n_{k}}(y)-2 \pi y\right| \geq \varepsilon, \quad k=1,2, \ldots .
$$

Then by Helly's Selection Theorem, there is a subsequence $\left(m_{k}\right)$ of $\left(n_{k}\right)$ such that

$$
\lim _{k \rightarrow \infty} F_{m_{k}}(y)=F(y)
$$

exists for every $x \in[0,1]$. Then Theorem 2.2, (4.18), (2.4), (2.5), and the Lebesgue Dominated Convergence Theorem imply that

$$
\int_{0}^{1} x^{q} d F(x)=\frac{1}{q+1}, \quad q=1,2, \ldots
$$

That is, all the corresponding moments of the measure $d F(x)$ and the function $x \rightarrow 2 \pi x$ are the same on $[0,1]$. Therefore, using the uniqueness part of the Riesz 
Representation Theorem describing all continuous linear functionals on $C[0,1]$, we obtain that $F(x)=2 \pi x$ for all $x \in[0,1]$. However, this contradicts (4.19) and (4.20). So

$$
\mathrm{m}\left\{t \in[0,2 \pi]: 0 \leq \alpha_{n}^{\prime}(t) \leq n x\right\}=2 \pi x+o_{n}(x)
$$

for every $x \in[0,1]$, where $\lim _{n \rightarrow \infty} o_{n}(x)=0$ for every $x \in[0,1]$.

To see the second statement of the theorem, we argue as follows. Using notation (2.1) and Lemma 4.2 we have $R_{n}^{\prime}(t)=o_{n}(t) n^{3 / 2}$ with a constant $o_{n}(t)$ tending to 0 as $n \rightarrow \infty$ for every $t \in \mathbb{R}$. Therefore

$\left|P_{n}^{\prime}\left(e^{i t}\right)\right|=\left|R_{n}^{\prime}(t) e^{i \alpha_{n}(t)}+i \alpha_{n}^{\prime}(t) e^{i \alpha_{n}(t)} R_{n}(t)\right|=o_{n}(t) n^{3 / 2}+\left|\alpha_{n}^{\prime}(t)\right|\left(1+\varepsilon_{n}(t)\right) \sqrt{n+1}$,

where $o_{n}(t)$ and $\varepsilon_{n}(t)$ tend to 0 as $n \rightarrow \infty$ for every $t \in \mathbb{R}$. Now the result follows from the first part of the theorem.

Proof of Theorem 2.2 for all real $q>0$. This follows from the already proved Conjecture 2.1 in a routine fashion.

Proof of Theorem 2.3. This follows immediately from Theorem 2.2, (3.5), and from the observation that

$$
\operatorname{Im}\left(\frac{e^{i t} P_{n}^{\prime}\left(e^{i t}\right)}{P_{n}\left(e^{i t}\right)}\right)=o_{n}(t)
$$

with suitable constants $o_{n}(t)$ such that $\max _{0 \leq t \leq 2 \pi}\left|o_{n}(t)\right|$ converge to 0 as $n \rightarrow \infty$. To see (4.21), we proceed as follows. Associated with a polynomial

$$
p_{n}(z)=\sum_{j=0}^{n} a_{j} z^{j}, \quad a_{j} \in \mathbb{C},
$$

we define

$$
\bar{p}_{n}(z)=\sum_{j=0}^{n} \bar{a}_{j} z^{j}
$$

Now let

$$
Q_{n}(t):=P_{n}\left(e^{i t}\right) \bar{P}_{n}\left(e^{-i t}\right)-(n+1)=\left|P_{n}\left(e^{i t}\right)\right|^{2}-(n+1),
$$

a trigonometric polynomial of degree $n$. Now $\left(P_{n}\right) \in \mathrm{UF}\left(\left(\varepsilon_{n}\right)\right)$ and (4.22) yield

$$
\left|Q_{n}(t)\right| \leq 3 \varepsilon_{n}(n+1),
$$

hence by Lemma 4.1 (Bernstein's Inequality for Trigonometric Polynomials), we obtain that

$$
\left|i e^{i t} P_{n}^{\prime}\left(e^{i t}\right) \bar{P}_{n}\left(e^{-i t}\right)-i e^{-i t} \bar{P}_{n}^{\prime}\left(e^{-i t}\right) P_{n}\left(e^{i t}\right)\right|=\left|Q_{n}^{\prime}(t)\right| \leq n 3 \varepsilon_{n}(n+1)
$$

for every $t \in \mathbb{R}$. Combining this with

$$
P_{n}\left(e^{i t}\right) \bar{P}_{n}\left(e^{-i t}\right)=\left|P_{n}\left(e^{i t}\right)\right|^{2}=n+\beta_{n}(t) n,
$$


where $\beta_{n}(t)$ are suitable constants such that $\max _{0 \leq t \leq 2 \pi}\left|\beta_{n}(t)\right|$ converge to 0 as $n \rightarrow \infty$, we conclude

$$
\begin{aligned}
\left|-2 \operatorname{Im}\left(\frac{e^{i t} P_{n}^{\prime}\left(e^{i t}\right)}{P_{n}\left(e^{i t}\right)}\right)\right| & =\left|i\left(\frac{e^{i t} P_{n}^{\prime}\left(e^{i t}\right)}{P_{n}\left(e^{i t}\right)}-\overline{\left(\frac{e^{i t} P_{n}^{\prime}\left(e^{i t}\right)}{P_{n}\left(e^{i t}\right)}\right)}\right)\right| \\
& =\left|\frac{i e^{i t} P_{n}^{\prime}\left(e^{i t}\right) \bar{P}_{n}\left(e^{-i t}\right)}{P_{n}\left(e^{i t}\right) \bar{P}_{n}\left(e^{-i t}\right)}-\frac{i e^{-i t} \bar{P}_{n}^{\prime}\left(e^{-i t}\right) P_{n}\left(e^{i t}\right)}{P_{n}\left(e^{i t}\right) \bar{P}_{n}\left(e^{-i t}\right)}\right| \\
& =\left|\frac{i e^{i t} P_{n}^{\prime}\left(e^{i t}\right) \bar{P}_{n}\left(e^{-i t}\right)-i e^{-i t} \bar{P}_{n}^{\prime}\left(e^{-i t}\right) P_{n}\left(e^{i t}\right)}{P_{n}\left(e^{i t}\right) \bar{P}_{n}\left(e^{-i t}\right)}\right| \\
& \leq \frac{n 3 \varepsilon_{n}(n+1)}{n+\beta_{n}(t)}=\beta_{n}^{*}(t) n,
\end{aligned}
$$

with suitable constants $\beta_{n}^{*}(t)$ such that $\max _{0 \leq t \leq 2 \pi}\left|\beta_{n}^{*}(t)\right|$ converge to 0 as $n \rightarrow \infty$. This proves (4.21), and hence the theorem is also proved.

Proof of Theorem 2.5. To see the second part of the theorem, we write, as in (2.1),

$$
P_{n}\left(e^{i t}\right)=R_{n}(t) e^{i \alpha_{n}(t)},
$$

where, as before, $R_{n}(t)=\left|P_{n}\left(e^{i t}\right)\right|$. Then

$$
P_{n}^{(r)}(z)=\sum_{k=0}^{r}\left(\begin{array}{l}
r \\
k
\end{array}\right) R_{n}^{(k)}(t) \frac{d^{(r-k)}}{d t^{r-k}}\left(e^{i \alpha_{n}(t)}\right)
$$

Now the theorem follows from (2.1), Theorem 2.4, Lemma 4.2, and Conjecture 2.1 .

\section{Remarks.}

If $Q_{n}$ is a polynomial of degree $n$ of the form

$$
Q_{n}(z)=\sum_{k=0}^{n} a_{k} z^{k}, \quad a_{k} \in \mathbb{C},
$$

and the coefficients $a_{k}$ of $Q_{n}$ satisfy

$$
a_{k}=\bar{a}_{n-k}, \quad k=0,1, \ldots n,
$$

then we call $Q_{n}$ a conjugate-reciprocal polynomial of degree $n$.

Remark 5.1. One can ask how flat a conjugate reciprocal unimodular polynomial can be. We present a simple result here. Let $P_{n} \in \mathcal{K}_{n}$ be a conjugate reciprocal polynomial of degree $n$. Then

$$
\max _{z \in \partial D}\left|P_{n}(z)\right| \geq(1+\varepsilon) \sqrt{n}
$$

with $\varepsilon:=\sqrt{\frac{4}{3}}-1$. This is an observation made by Erdös [Er2] but his constant $\varepsilon>0$ is unspecified. 
To prove the statement, observe that Malik's inequality [MMR, p. 676] gives

$$
\max _{z \in \partial D}\left|P_{n}^{\prime}(z)\right| \leq \frac{n}{2} \max _{z \in \partial D}\left|P_{n}(z)\right|
$$

(Note that the fact that $P_{n}$ is conjugate reciprocal improves the Bernstein factor on $\partial D$ from $n$ to $n / 2$.) Using $P_{n} \in \mathcal{K}_{n}$, Parseval's formula, and Malik's inequality, we obtain

$$
2 \pi \frac{n^{3}}{3} \leq 2 \pi \frac{n(n+1)(2 n+1)}{6}=\int_{\partial D}\left|P_{n}^{\prime}(z)\right|^{2}|d z| \leq 2 \pi\left(\frac{n}{2}\right)^{2} \max _{z \in \partial D}\left|P_{n}(z)\right|^{2},
$$

and

$$
\max _{z \in \partial D}\left|P_{n}(z)\right| \geq \sqrt{4 / 3} \sqrt{n}
$$

follows.

Remark 5.2. Assume that $\left(P_{n}\right)$ is an ultraflat sequence of unimodular polynomials $P_{n} \in \mathcal{K}_{n}$. As before, we use notation (2.1). We denote the number of zeros of $P_{n}$ inside the open unit disk $D$ by $Z\left(P_{n}\right)$. We claim that

$$
Z\left(P_{n}\right)=\frac{n}{2}\left(1+o_{n}\right),
$$

where $o_{n}$ is a sequence converging to 0 as $n \rightarrow \infty$. To see this we argue as follows. By Conjecture 2.1 (that we proved) we have

$$
\alpha_{n}(2 \pi)-\alpha_{n}(0)=\frac{1}{2}\left(1+o_{n}\right)(2 \pi)=\left(1+o_{n}\right) n \pi
$$

with constants $o_{n}$ converging to 0 as $n \rightarrow \infty$. So the "Argument Principle" yields the result we stated.

\section{Acknowledgment.}

I thank Peter Borwein for sending me Saffari's paper [Sa] and for many discussions related to the topic.

\section{REFERENCES}

[Be] J. Beck, "Flat" polynomials on the unit circle - note on a problem of Littlewood, Bull. London Math. Soc. (1991), 269-277.

[BE] P. Borwein and T. Erdélyi, Polynomials and Polynomial Inequalities, Springer-Verlag, New York, 1995.

[By] J.S. Byrnes, On polynomials with coefficients of modulus one, Bull London Math. Soc. 9 (1977), 171-176.

[BEK] P. Borwein, T. Erdélyi, and G. Kós, Littlewood-type problems on [0,1], Proc. London Math. Soc. (3) 79 (1999), 22-46. 
[BS] J.S. Byrnes and B. Saffari, Unimodular polynomials: a wrong theorem with very fortunate consequences, Bull London Math. Soc. (to appear).

[DL] R.A. DeVore and G.G. Lorentz, Constructive Approximation, Springer-Verlag, Berlin, 1993.

[Er1] P. Erdős, Some unsolved problems, Michigan Math. J. 4 (1957), 291-300 Berlin.

[Er2] P. Erdős, An inequality for the maximum of trigonometric polynomials, Annales Polonica Math. 12 (1962), 151-154.

[Ka] J.P. Kahane, Sur les polynomes a coefficient unimodulaires, Bull. London Math. Soc. 12 (1980), 321-342.

[Kö] T. Körner, On a polynomial of J.S. Byrnes, Bull. London Math. Soc. 12 (1980), 219224.

[Li1] J.E. Littlewood, On polynomials $\sum \pm z^{m}, \sum \exp \left(\alpha_{m} i\right) z^{m}, z=e^{i \theta}$, J. London Math. Soc. 41 (1966), 367-376.

[Li2] J.E. Littlewood, Some Problems in Real and Complex Analysis, Heath Mathematical Monographs, Lexington, Massachusetts, 1968.

[MMR] Milovanović, G.V., D.S. Mitrinović, \& Th.M. Rassias, Topics in Polynomials: Extremal Problems, Inequalities, Zeros, World Scientific, Singapore, 1994.

[QS1] H. Queffelec and B. Saffari, Unimodular polynomials and Bernstein's inequalities, C. R. Acad. Sci. Paris Sér. I Math. 321 (1995, 3), 513-318.

[QS2] H. Queffelec and B. Saffari, On Bernstein's inequality and Kahane's ultraflat polynomials, J. Fourier Anal. Appl. 2 (1996, 6), 519-582.

[Sa] B. Saffari, The phase behavior of ultraflat unimodular polynomials, in Probabilistic and Stochastic Methods in Analysis, with Applications (1992), Kluwer Academic Publishers, Printed in the Netherlands.

Department of Mathematics, Texas A\&M University, College Station, Texas 77843

E-mail address: terdelyi@math.tamu.edu 